\title{
Determination of Media Attributes Through Fuzzy Analytical Hierarchy Process and Artificial Neural Network Model
}

\author{
Maryam Haghshenas* \\ Media Management, University of Tehran, Iran
}

\section{ABSTRACT}

By identifying the needs and demands of the media audience, we can try to include them at all stages of the design and production of the media, thus helping the media industry succeed and provide the user with the satisfaction of the desired product. They provided it and created a user satisfaction by presenting the product in accordance with its requirements. Based on this approach, media users typically present their mental needs in their initial assessments of the presentation of a media product, and then these objective needs systematically and using a wide range of different methods and tools are transmitted to the media product designer. In this regard, decision-making systems play a significant role in the development of the media industry and help media users in the correct and efficient use of media. In this paper, with the goal of designing a decision support system, the general needs and attributes of the media audience have been identified. Then, weights and values of attributes and alternatives are determined by the fuzzy analytical hierarchy process method by media experts. The purpose of the designed model is that decision makers using neural networks and knowing the rank of the media determine the attributes of the audience selected media (for example, TV, Newspaper, Radio, andFacebook), based on the needs of the audience in line with the goals of the studied media organization and help the audience to choose the media.

Key words: Artificial neural network, decision support systems, fuzzy analytical hierarchy process, media, organizations

\section{INTRODUCTION}

McLuhan, Canadian professor, philosopher, and public intellectual is known for coining the expression "the medium is the message" and the term global village. He believed that technology was not neutral and that any kind of technology had various consequences and effects. Therefore, ICTs have more significant outcomes and effects. ${ }^{[1]}$ Media is the collective communication outlets or tools that are used to store and deliver information or data. It is either associated with communication media or the specialized mass media communication businesses such as print media and the press, photography, advertising, cinema, broadcasting (radio and television), and publishing and point of sale (Wikipedia). ${ }^{[2]}$ Media technology, as one of the most influential factors, has been blended with the lives of people in various societies. By ignoring it, it will create a major disorder in society and its various social, cultural, political, and economic dimensions. ${ }^{[1]}$ According to another definition, the media is the means by which the message is presented. In other words, media are tools used to store and deliver information or data and have different types, for example, visual media (TV), written media

${ }^{*}$ Corresponding author:

Email: M_haghshenas@ut.ac.ir

http://dx.doi.org/10.20530/EJIBM410

ISSN 2056-9904 ๔ 2017 (newspapers), audio media (radio), and social media (Facebook). ${ }^{[3]}$

One of the important issues in media technology is the choice of media. In fact, the choice of a medium must be made in accordance with certain principles, to select a suitable medium with unique characteristics. No media, device, procedure, or experience alone can be the best for certain goals, and you need to make sure that the chosen media is consistent with goals. The effective use of media in a particular subject requires an adequate knowledge of the media. The selected media should be appropriate to the needs of the audience. Personal interests should not be affected by the choice of media. A media for a specific purpose can't be excellent for other purposes. For example, a media or an excellent educational aid may not result in a poorly educated learning environment. Hence, experiences, inclinations, abilities, and types of learners' learning approach may also have an impact on the outcomes of using media and teaching aids. There are a lot of factors affecting the selection of media. Some of them are:

- Position of use

- Type of media

- Audience features

- Usage Environment

- Terms of development

- Accessibility

- Acceptability

- Costs. 


\section{PROPOSED MODEL}

The structure of the proposed model is shown in Figure 1. The required data are initially prepared and make the comparison Matrix. The Matrix is made up of decision maker's (DM) information on criteria and alternatives. The results are used to design, train, and after that to simulate the artificial neural network (ANN) model. This ANN model is learning the machine. It trained with replacing input and output of fuzzy analytical hierarchy process (AHP) model. It is an able DM to achieve weights of properties by input alternatives ranking. The approval of these results and a final decision is made by the DM.

The hierarchy construction used in the model is shown in Figure 2. Figure 2 shows the hierarchy diagram of the main criteria and criteria used to select Media. At the top level (Level 1), the goal of the model is defined which is to select the most suitable media among the alternatives at the bottom level, assigned from a database created for this purpose. To achieve this objective, a number of main criteria (Level 2) and criteria (Level 3) are defined in Figure 2.

\section{Fuzzy AHP (FAHP)}

In the conventional AHP method first is developed by Saaty. Pairwise comparisons for each level with respect to the goal of the best alternative selection are conducted using a nine-point scale. Different types of fuzzy membership functions have been used in fuzzy logic. However, three types are most common: Monotonic, triangular, and trapezoidal. Because the fuzzy set is a convex function, the trapezoidal function

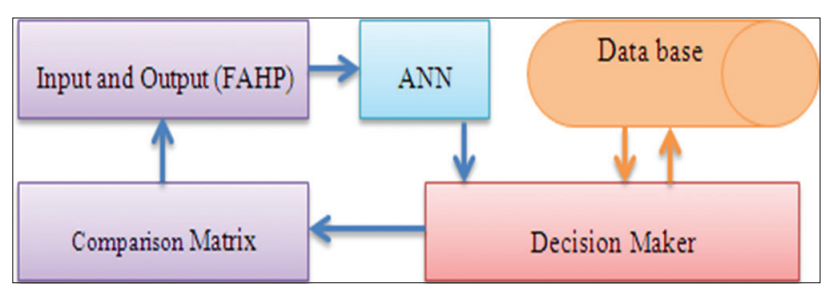

Figure 1: Proposed model

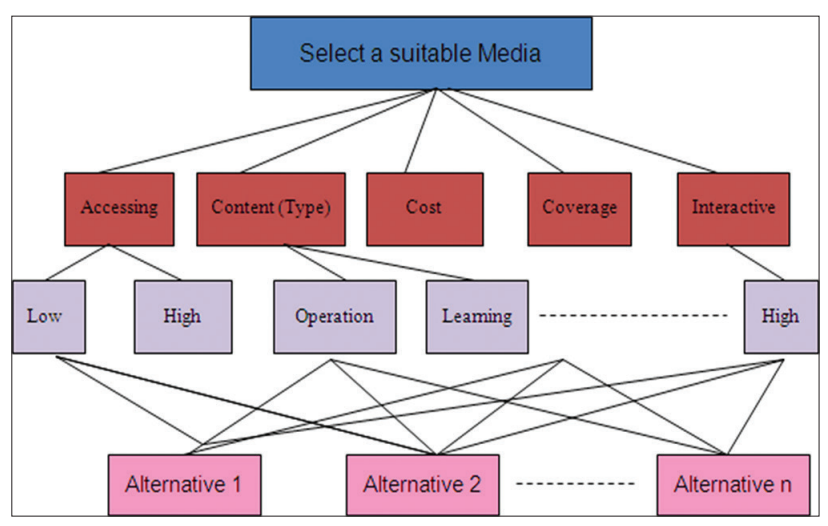

Figure 2: Hierarchy structure or triangular function approaches the convex function well. The triangular fuzzy numbers are more convenient in applications due to their computational simplicity, and they are useful in promoting representation and information processing in a fuzzy environment. ${ }^{[4]}$

The characteristics and membership function of the triangular fuzzy number are expressed by Eq. (1):

$\mu(x)=\left\{\begin{array}{cc}(x-1) /(m-1) & , 1 \leq x \leq m \\ (u-x) /(u-m) & , m \leq x \leq u \\ 0, & \text { Otherwise }\end{array}\right.$

By introducing the a-cut and defining the interval of confidence at confidence level $\alpha$, the triangular fuzzy number can be characterized by Eq. (2). The $\alpha$-cut is known to incorporate the experts or $\mathrm{DM}(\mathrm{s})$ confidence over his/ her preference or the judgments.

$$
\forall \alpha \int[0,1] \tilde{\mathrm{A}}_{\mathrm{a}}=\left[{ }_{1}{ }_{\omega} \mu_{\mathrm{a}}\right]=[(\mathrm{m}-1) \mathrm{a}+1, \mu-(\mu-\mathrm{m}) \mathrm{a}]
$$

The degree of satisfaction can be estimated from the DM by the index of optimism $\lambda$ by Eq. (3), where its value range is $0<\lambda<1$. The larger the index $\lambda$, the higher the degree of satisfaction:

$\tilde{\mathrm{a}}_{\mathrm{ij}}^{\mathrm{a}}=\lambda \mathrm{a}^{\mathrm{a}}{ }_{\mathrm{iju}}+(1-\lambda) \mathrm{a}^{\mathrm{a}}{ }_{\mathrm{ijl}}, \forall \lambda \varepsilon[0,1]$

The matrix $\widetilde{A}$ is reconstructed $\tilde{a}_{\mathrm{ij}}^{a}$ using the equation above, and the degree of satisfaction can be estimated setting the index of optimism $\lambda$ and fixing $\alpha$. Therefore:

$$
\tilde{\mathrm{A}}=\left[\begin{array}{ccccc} 
& & & & \\
& & & & \\
1 & \tilde{a}_{12}^{a} & \ldots & \ldots & \tilde{a}_{12}^{a} \\
\tilde{a}_{21}^{a} & 1 & \ldots & \ldots & \tilde{a}_{2 n}^{a} \\
\cdot & \cdot & \ldots & \ldots & \cdot \\
\cdot & \cdot & \ldots & \ldots & \cdot \\
\tilde{a}_{n 1}^{a} & \tilde{a}_{n 2}^{a} & \ldots & \ldots & 1
\end{array}\right]
$$

The five triangular fuzzy numbers and their reciprocal scale are defined with the corresponding membership function as shown in Table 1.

Calculating the overall priority weight for each alternative (AW) by multiplying the vector of criteria weight $(\mathrm{CW})$ by the matrix of alternative evaluation weights using the equation below:

$$
A W_{k}=\sum_{\mathrm{i}=1}^{\mathrm{n}} C W_{i}+A E W_{i k}
$$

Where $n=$ Number of criteria, $m=$ Number of alternatives, and $k=1,2, \ldots, m$.

To identify the consistency ratio (CR) of a matrix, first, the matrix consistency index $(\mathrm{CI})$ is found by: 
Table 1: Definition and membership functions of fuzzy numbers

\begin{tabular}{|c|c|c|c|}
\hline $\begin{array}{l}\text { Fuzzy } \\
\text { numbers }\end{array}$ & Definition & $\begin{array}{l}\text { Membership } \\
\text { function }\end{array}$ & $\begin{array}{l}\text { Reciprocal } \\
\text { scale }\end{array}$ \\
\hline 1 & Equally important & $(1,1,2)$ & $(1 / 2,1,1)$ \\
\hline$\tilde{3}$ & Moderately important & $(2,3,4)$ & $(1 / 4,1 / 3,1 / 2)$ \\
\hline$\tilde{5}$ & Strongly important & $(4,5,6)$ & $(1 / 6,1 / 5,1 / 4)$ \\
\hline$\tilde{7}$ & Very Strongly important & $(6,7,8)$ & $(1 / 8,1 / 7,1 / 6)$ \\
\hline$\tilde{9}$ & Extremely important & $(8,9,10)$ & $(1 / 10,1 / 9,1 / 8)$ \\
\hline$\tilde{2}, \tilde{4}, \tilde{6}, \tilde{8}$ & Intermediate values & & \\
\hline
\end{tabular}

$C I=\left(\lambda_{\max }\right)+(n-1)$

The CI of a randomly generated reciprocal matrix with reciprocal forces is called the random index (RI) and is calculated using the matrix order ( $n$ ) and the table explained by Saaty.

Hence, the matrix CR is calculated using:

\section{$C R=C I / R I$}

A consistency ratio of 0.1 or less is considered acceptable. Then, compute geometric mean of weights of FAHP for group decision-making. To find the weights of the relationship matrix by the FAHP model, a program is developed using MATLAB.

This paper used AHP fuzzy for selection suitable media. This process helps to be success media industrial and get satisfaction of user with media alternative according to demands. Media users say own intellectual needs by basic evaluations about product and FAHP systematically use different methods and instruments to rank these needs to the actual alternative.

\section{ANN}

The ANN are known as the "universal approximates" and "computational models" with particular characteristics such as the ability to learn or adapt, to organize or to generalize data. ${ }^{[5]}$ Up-to-date designing a (near) optimal network architecture is made by a human expert and requires a tedious trial and error process. On the other hand, they are simplified mathematical approximations of biological neural networks in terms of structure as well as function.

ANNs can be classified into two major categories: Supervised and unsupervised ANNs. ${ }^{[6]}$ In supervised ANNs, there is usually a DM who can provide some feedback in terms of evaluating the given set of training patterns, while the unsupervised ANNs do not require the external evaluator. Supervised learning systems are generally more flexible in the design of hidden layers. ANN architecture is generally described as an arrangement of interconnected nodes organized into three groups input, hidden, and output. The most commonly used approach to ANN learning is the feed-forward backpropagation algorithm.

A feed-forward ANN consists of an input layer, an output layer, and a variable number of hidden layers. ${ }^{[7]}$ The input layer is not counted normally, because it is only formally present, in the sense that it does not do any processing: For example, a two-layer network consists of the input, hidden, and output layers. All connections between the layers are allowed. Connections between the nodes of the same layer, as well as the auto connections (loops), are prohibited. Using backpropagation algorithm, the weights in the networks are adjusted in any iteration as to reduce the error.

The backpropagation algorithm may be described with the following three steps, which have to be applied several times in iteration. ${ }^{[5]}$

1. Forward computation of input signal of training sample and determination of neural network response.

2. Computation of an error between desired response and neural network response.

3. Backward computation of the error and calculation of corrections to synaptic weights and biases.

The designed ANN consists of three layers: An input layer, a hidden layer, and an output layer. In this paper, a supervised feed-forward backpropagation ANN is designed, and values from FAHP Model, where the importance of attributes are determined. ANN Input is output of FAHP that show ranking of alternatives and set output same as input of score of criteria [Figure 2].

The algorithm Levenberg-Marquardt back propagation (trainlm) is used to design network model for training in MATLAB $^{\circledR}$ software. This algorithm appears to be the fastest method for training moderate-sized feed-forward neural networks (up to several hundredweights). It also has an efficient implementation in MATLAB ${ }^{\circledR}$ software, because the solution of the matrix equation is a built-in function, so its attributes become even more pronounced in a MATLAB environment.

10 input-output sets (10 DMs) mentioned above are then split into two parts: part one (7 sets) is used for training the neural network and the second part ( 3 sets) where they are not used in training stage, is used to test the ANN model. At the end, results from the ANN model are then compared with FAHP model.

In the proposed model, the ANN model is used to verify the results of the FAHP model and to predict the importance of criteria. On the other hand, once the neural network is trained, it can be used to predict the criteria ranking with any input-output set of judgments from DM(s). The ANN is applied using MATLAB 7.4 (R2007a) software. 


\section{IMPLEMENTED MODEL}

In this section, an algorithm is proposed, which can be divided into two major phases. In the first phase, the comparison matrix is constructed with using FAHP approach, in the second phase, the results of the first phase used for design and train ANN approach for finding media properties.

\section{Stage I: Pairwise Comparisons Matrixes and Calculation with FAHP}

The basic accepted main criteria shown in Table 2 . These criteria are extracted from reviewed literature and interviewed with experts of media. According to the most chosen items, the following results to determine the characteristics were obtained. These features are presented in level 1 and level 2 of the hierarchy structure.

A questionnaire was developed. Respondents were asked to express their views on using media for learning, news and fun purposes. Users must provide their level of equally/ extremely important using the scale from 0 to 6 . Table 3 is shown one matrix that fill from answers. Furthermore, user responds the scale 1-6 for pairwise comparisons matrix of criteria filled by fuzzy numbers and normalized. Tables 3 and 4 are shown in first DM's judgment. The next step for the DMs is assigning the preference score for alternatives with respect to each criteria, Tables 5 and 6 are shown results of first DM with respect Accessing low media.

Table 2: Criteria for selection Media

\begin{tabular}{ll} 
Accessing & Low $(\mathrm{AL})$, High $(\mathrm{AH})$ \\
Content (Type) & Practical (CP), Learning (CLG) \\
Cost & Low (CL), Medium (CM), High (CH) \\
Coverage & Limited (CLD), Wide (CW) \\
Interactive & Low (IL), High (IH) \\
\hline
\end{tabular}

\section{Stage II: Train and Test ANN Model}

The results from FAHP Model are used to design and train the proposed ANN model. The next steps are to find the CWs and ranking using the ANN model as follows:

Step 1: Four alternative weights of $10 \mathrm{DM}$ are used for input values of the ANN model and the relative weight total of the $11 \mathrm{CWs}$ are used as a target.

Step 2: Designing ANN model using Levenberg-Marquardt back propagation algorithm with a different number of hidden nodes, 7 , and 10 .

Step 3: Training the model with same training parameters, learning rate and validation rate using different of epoch [Table 7]. The mean square error value is used as the stop criteria.

Step 4: Use 7 samples for training and 3 samples for testing.

The used models have been achieved with the trainlm training function. See Figures 3 and 4 the best performance Net1 and Net2. The degree of closeness in training data in much higher than testing and evaluating data, and it shows the accuracy of the designed network.

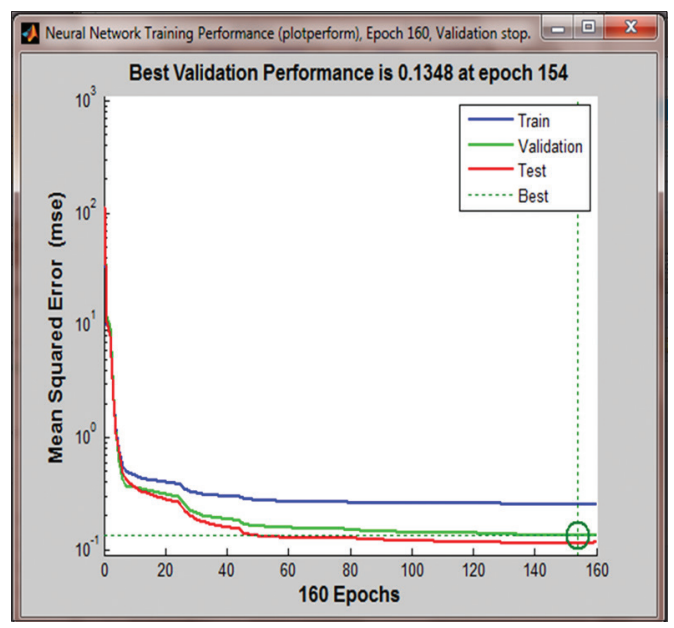

Figure 3: Performance of Net1 for (4-7-11) model

Table 3: Normalize comparison matrix for the criteria (DM1)

\begin{tabular}{lccccccccccccccc}
\hline & AL & AH & CP & CLG & CL & CM & CH & CLD & CW & IL & 0.98 & 0.655 & 0.989 \\
\hline AL & 0.706 & 0.28 & 0.192 & 0.45 & 0.798 & 0.926 & 0.087 & 0.95 & 0.98 \\
AH & 0.434 & 0.699 & 0.527 & 0.243 & 0.502 & 0.282 & 0.679 & 0.868 & 0.414 & 0.157 & 0.461 \\
CP & 0.042 & 0.287 & 0.604 & 0.999 & 0.908 & 0.91 & 0.462 & 0.725 & 0.334 & 0.858 & 0.707 \\
CLG & 0.487 & 0.006 & 0.804 & 0.67 & 0.31 & 0.413 & 0.675 & 0.232 & 0.273 & 0.672 & 0.913 \\
CL & 0.264 & 0.614 & 0.244 & 0.237 & 0.692 & 0.485 & 0.403 & 0.545 & 0.227 & 0.224 & 0.365 \\
CM & 0.93 & 0.83 & 0.921 & 0.446 & 0.845 & 0.862 & 0.574 & 0.144 & 0.443 & 0.402 & 0.248 \\
CH & 0.759 & 0.189 & 0.461 & 0.712 & 0.66 & 0.655 & 0.79 & 0.336 & 0.109 & 0.293 & 0.728 \\
CLD & 0.286 & 0.572 & 0.197 & 0.052 & 0.213 & 0.337 & 0.402 & 0.47 & 0.138 & 0.816 & 0.838 \\
CW & 0.966 & 0.05 & 0.821 & 0.06 & 0.777 & 0.051 & 0.48 & 0.295 & 0.202 & 0.564 & 0.751 \\
IL & 0.589 & 0.066 & 0.4 & 0.217 & 0.449 & 0.582 & 0.564 & 0.376 & 0.346 & 0.566 & 0.787 \\
\hline
\end{tabular}

Table 4: The criteria weights for first decision maker (DM1)

\begin{tabular}{lccccccccccc}
\hline AL & AH & CP & CLG & CL & CM & CH & CLD & CW & IL & IH \\
\hline 0.546 & 0.359 & 0.517 & 0.409 & 0.615 & 0.55 & 0.512 & 0.494 & 0.347 & 0.521 & 0.679 \\
\hline
\end{tabular}


The comparison of the outputs from the FAHP model and ANN is made for one DM, and the result is shown in Table 8. One can obviously observe, the ranking of the criteria for both FAHP model and ANN model are the same. Hence, the proposed decision support system by combining the FAHP and ANN in this work can be used as an active tool to know criteria score according score of media to satisfy user needs.

In this study, the fuzzy model was verified and compared with a developed ANN model. As the number of training data samples is increased, the ANN

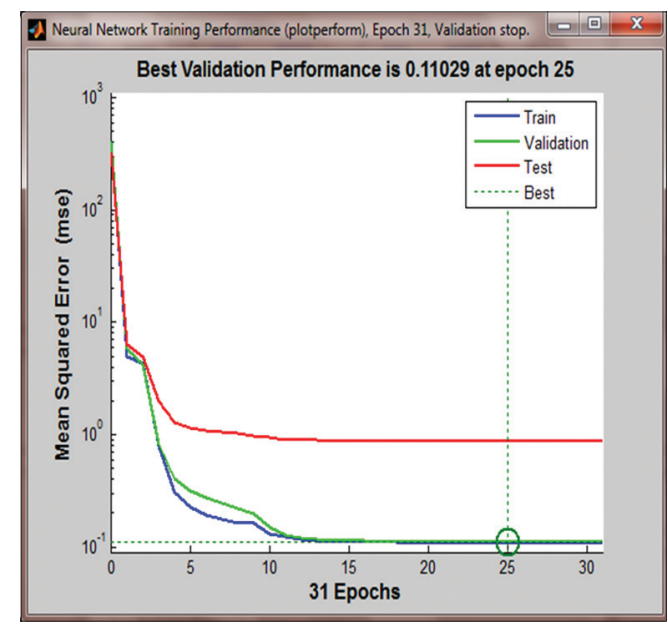

Figure 4: Performance of Net2 for (4-10-11) model

Table 5: Normalize comparison matrix of alternatives for accessing low $(A L)$

\begin{tabular}{lcccc}
\hline (AL) & TV & Newspaper & Radio & Facebook \\
\hline TV & 0.045 & 0.162 & 0.574 & 0.144 \\
Newspaper & 0.66 & 0.605 & 0.19 & 0.336 \\
Radio & 0.013 & 0.337 & 0.402 & 0.47 \\
Facebook & 0.077 & 0.051 & 0.48 & 0.195 \\
\hline
\end{tabular}

Table 6: The alternatives weights of first decision maker (DM1) for accessing low (AL)

\begin{tabular}{ccccc}
\hline & TV & Newspaper & Radio & Facebook \\
\hline (AL) & 0.199 & 0.289 & 0.412 & 0.286 \\
\hline
\end{tabular}

model can train faster and learn the selection problem very well.

Furthermore, once the network is trained, it can be used for predicting CWs by either team decision-making or single DM saving time and effort for the new decision-making process.

\section{CONCLUSION}

The dynamic and changing organizations that have advanced in the field of computer technology have created a potential portfolio of DSS applications. However, with the advancement of information technology and process change consumption patterns, product lifecycle is shorter and shorter. Hence, the development of current products is the main strategy of organizations, and to create customer satisfaction, new products must be developed based on customer needs. The media organization is no exception to this, and it must take steps toward the advancement of technology in the era of technology, and, to satisfy the audience, to provide media products in accordance with their needs and desires. For this reason, in this research, DSS was used as a tool for designing and determining attributes of media in the media industry to reduce product development costs and improve quality.

Based on the research model, using the neural network and fuzzy logic, first, the general attributes of the media are considered by media professionals and experts and ranked with the help of a FAHP technique. Then the neural network, using the rank of each medium, determines the attributes or properties of media for DM so that they can help audiences based on their needs for media selection. It should be noted that by constantly changing the characteristics and media of the target audience, the neural network must be educated and tested with outputs of FAHP technology and provide optimal results.

Table 7: ANN model parameters

\begin{tabular}{|c|c|c|c|c|c|c|c|c|c|}
\hline & Input nodes & Hidden nodes & Output nodes & $\begin{array}{l}\text { Training } \\
\text { function }\end{array}$ & Learning rate & $\begin{array}{c}\text { Validation } \\
\text { rate }\end{array}$ & Test rate & Epoches & Performance \\
\hline 1 & 4 & 7 & 11 & Trainlm & 0.7 & 0.15 & 0.15 & 160 & 0.1348 \\
\hline 2 & 4 & 10 & 11 & Trainlm & 0.7 & 0.15 & 0.15 & 31 & 0.11029 \\
\hline
\end{tabular}

ANN: Artificial neural network

Table 8: Comparison between fuzzy AHP and ANN models (criteria weight)

\begin{tabular}{|c|c|c|c|c|c|c|c|c|c|c|c|}
\hline & $A L$ & $\mathbf{A H}$ & $\mathrm{CP}$ & CLG & $\mathrm{CL}$ & CM & $\mathrm{CH}$ & CLD & CW & IL & IH \\
\hline Fuzzy AHP & 0.231 & 0.432 & 0.127 & 0.502 & 0.415 & 0.512 & 0.354 & 0.314 & 0.517 & 0.319 & 0.231 \\
\hline ANN models (4-7-11) & 0.243 & 0.439 & 0.125 & 0.505 & 0.409 & 0.516 & 0.358 & 0.319 & 0.519 & 0.318 & 0.236 \\
\hline ANN models (4-10-11) & 0.251 & 0.425 & 0.131 & 0.515 & 0.413 & 0.521 & 0.359 & 0.312 & 0.516 & 0.319 & 0.243 \\
\hline
\end{tabular}




\section{REFERENCES}

1. Ghahramani AR. Investigating the Influence of the Media on the Recognition and Persuasion of the Audience, Published in the Proceedings of the First National Conference on Modern Management Science and Social Cultural Planning; 2015.

2. Available from: https://www.wikipedia.org. [Last accessed on 2017 Oct 15].

3. Zarezadeh F, Moghaddam HS. Social Media in the Service of Education and Research in the New World of Information, Published in the Proceedings of the First International Media Literacy Conference in Iran, October, 30; 2014.
4. Jeddi YP. A fuzzy decision system for new product specification forecast. Int $J$ Bus Manage Soc Res 2016;2:105-13.

5. Rady HA. Shannon entropy and mean square errors for speeding the convergence of multilayer neural networks: A comparative approach. Egypt Inf J 2011;12:197-209.

6. Taha Z, Rostam S. A fuzzy AHP-ANN-based decision support system for machine tool selection in a flexible manufacturing cell. Int J Adv Manuf Technol 2011;57:719-33.

7. Vishwa A, Alka V, Sharma A. Pre-diagnosis of lung cancer using feed forward neural network and back propagation algorithm. Int J Comput Sci Eng 2011;3:3313-9. 